\title{
Un enseignement structuré du lexique dès la maternelle au service de l'écriture
}

Marie-Noëlle Roubaud et Marie-José Moussu

\section{(2) OpenEdition}

12 Journals

Édition électronique

URL : http://journals.openedition.org/pratiques/3484

DOI : 10.4000/pratiques.3484

ISSN : 2425-2042

Éditeur

Centre de recherche sur les médiations (CREM)

Édition imprimée

Date de publication : 15 décembre 2012

Pagination : 109-126

Référence électronique

Marie-Noëlle Roubaud et Marie-José Moussu, « Un enseignement structuré du lexique dès la maternelle au service de l'écriture », Pratiques [En ligne], 155-156 | 2012, mis en ligne le 18 décembre 2017, consulté le 14 novembre 2019. URL : http://journals.openedition.org/pratiques/3484 ; DOI : 10.4000/pratiques.3484 


\title{
Un enseignement structuré du lexique dès la maternelle au service de l'écriture
}

\author{
Marie-Noëlle Roubaud \\ Marie-José Moussu
}

EA 4671 ADEF

Aix-Marseille Université

ENS de Lyon, IFE

\section{Introduction}

Comme l'indiquent les travaux des chercheurs dans différents domaines (psychologie, sociologie, linguistique et didactique), un apprentissage informel du lexique ne suffit pas à développer des compétences de locuteur-scripteur. Déjà en 1993, Florin rappelait que la maîtrise du lexique ne relevait pas de l'imprégnation spontanée. Encore aujourd'hui, pour de nombreux enseignants, il semble que l'apprentissage du lexique se fasse de façon naturelle, en situation, par imprégnation. Même s'ils se plaignent des carences lexicales de leurs élèves tant dans leur production écrite qu'orale, dans leurs classes les activités sur le lexique ont le plus souvent un caractère non prévisible et non programmé. Elles se centrent essentiellement sur la relation lexique-orthographe, sur la définition du mot (Dreyfus, 2004) ou encore sont annexées à d'autres apprentissages considérés comme plus fondamentaux (David, 2000). Les enseignants organisent peu d'activités lexicales structurées dans une perspective de production de texte (Jousse et al., 2008: 154). L'enseignement du lexique est souvent morcelé et peu productif (Calaque, 2004), voire fossilisé.

L'enjeu d'une acquisition lexicale est important pour la maîtrise des textes en réception et en production et pour la réussite scolaire en général (Lieury, 2003). Cette acquisition est liée aux tâches d'écriture et « la mise en synergie » (Boch, 2004) de ces deux activités (apprentissage du lexique et production de texte) améliore à la fois l'acquisition lexicale et la qualité littéraire de la production (Duvignau et Garcia-Debanc, 2008). De ce fait, nous pensons qu'un apprentissage plus systématique et plus précoce est nécessaire. 
L'école a un rôle important à jouer dans le domaine de l'acquisition du lexique. Les programmes pour l'école de 2008 invitent les enseignants, dès l'école maternelle ${ }^{(1)}$, à réfléchir sur l'enseignement du vocabulaire et rappellent qu'une simple exposition de mots ne suffit pas à les mémoriser. Ils préconisent un enseignement plus systématique : des séquences spécifiques, des activités régulières de classification, de mémorisation de mots, etc. et le passage par l'écrit, la copie pour retenir les mots nouveaux. Ils laissent à l'enseignant le soin d'inventer et de mettre en œuvre des situations pédagogiques adéquates.

C'est pour répondre aux sollicitations des enseignants de maternelle, quelquefois démunis, que nous avons conçu et mis en œuvre un dispositif d'enseignement du lexique construit autour de quatre phases : observation, délibération, manipulation et structuration. Notre objectif était de les accompagner dans ce projet d'amélioration du lexique de leurs élèves. Nous avons fait l'hypothèse qu'un enseignement progressif et intégrateur du lexique dès l'école maternelle contribuait à une meilleure maîtrise du lexique et à une entrée facilitée dans l'écriture, c'est-à-dire au développement de compétences de lecteur-scripteur.

La première partie interroge les recherches scientifiques ayant porté sur le lexique. La seconde est une analyse des instructions officielles pour l'école. La troisième partie présente les principes d'un dispositif expérimental d'enseignement du lexique, né de notre réflexion théorique et institutionnelle. La dernière partie rend compte de l'expérimentation dans quatre classes de grande section de maternelle et des effets du dispositif sur les élèves en termes d'acquisitions et de comportements.

\section{La question du lexique du côté de la recherche}

\subsection{Lexique et catégorisation}

L'acquisition du lexique ${ }^{(2)}$ ne se laisse pas aisément décrire car comme l'écrit David (2000 : 34 et 36), il faut la concevoir comme « un réseau d'interactions ajustées entre des opérations cognitives générales et des fonctionnements linguistiques particuliers ». Cette acquisition va de pair avec l'organisation du système conceptuel de l'enfant (Florin, 2002:13): «[...] plus le système conceptuel de l'enfant est riche, tant du point de vue de son contenu que de son organisation, plus facile est l'activation en mémoire des objets. Or, pour catégoriser, il faut, entre autres, pouvoir se représenter en mémoire les propriétés des objets pour ensuite établir des relations entre plusieurs propriétés d'objets, de manière à dégager un trait commun $»$.

Florin (1993 : 99) propose de développer ce système conceptuel dans deux directions : du côté des structures catégorielles plus générales et abstraites et du côté des structures empiriques plus fines et plus complexes. Pour illustrer ces

(1) En France, l'école maternelle regroupe les enfants de 3 à 6 ans dans trois classes : petite section (3-4 ans), moyenne section (4-5 ans) et grande section (5-6 ans).

(2) Nous empruntons à Picoche (1992: 45) la définition du mot « lexique » distinguée de celle de «vocabulaire»: "On conviendra d'appeler lexique l'ensemble des mots qu'une langue met à la disposition des locuteurs, et vocabulaire l'ensemble des mots utilisés par un locuteur donné dans des circonstances données ». 
deux types de structures, donnons deux exemples, empruntés à Florin (1993: 97) : « le beurre c'est un aliment, c'est comme de l'huile» est une définition catégorielle qui met en relation les caractéristiques communes et distinctives d'objets et « on grimpe sur l' arbre pour aller chercher des fruits » est une définition de type empirique qui donne un exemple de l'utilisation du mot grimper. Lors de son expérimentation sur des élèves de 7 à 11 ans, Florin a constaté que les répertoires verbaux des enfants étaient d'autant plus étendus qu'ils appartenaient à des milieux socio-économiques favorisés et que ces différences subsistaient du CE1 au CM2. Elle a aussi identifié que les définitions catégorielles étaient plus fréquentes chez les enfants de milieux socio-économiques favorisés que chez les autres et qu'on observait le résultat inverse pour les définitions de type empirique (1993: 98).

\subsection{Lexique et activités métalinguistiques}

Florin (1993 : 100) montre, à la suite de Gombert (1990), que la maîtrise métalinguistique des mots est très décalée par rapport à leur utilisation, ce qui a des incidences nombreuses sur les différences entre milieux sociaux. A la suite de Lahire (1993), Branca-Rosoff et al. (2008 : 255) nous rappellent que les activités métalinguistiques sont particulièrement discriminantes pour les enfants des classes populaires. Pour développer une conscience métalinguistique chez les élèves, ces auteurs (2008 : 266) proposent d'installer des moments réguliers d'observation réfléchie de la langue, bien déconnectés du flux de la communication afin de leur donner une certaine habitude du " regard métalinguistique » puis de revenir à des activités discursives. Jousse et al. (2008: 154) soulignent également que cet enseignement du lexique devrait être conduit dans la perspective « d'une sensibilisation à la paraphrase comme moyen linguistique pour encoder la pensée $»$.

\subsection{Lexique et activités scolaires}

Les travaux de Picoche (1992: 47), catégorisant les mots de la langue en «mots actifs » ${ }^{(3)}$ et « mots passifs » ${ }^{(4)}$ amènent à penser un décalage entre la réception du lexique (compréhension) et sa production. Ainsi certains chercheurs proposent de découvrir les mots dans des situations diverses : lors d'activités, en lecture, en écriture, en orthographe. Si le nombre de confrontations nécessaires pour qu'un mot soit enregistré dans la mémoire est inconnu, Fayol affirme (2006: 61) que « la fréquence de rencontre a un impact très fort». De plus Florin (2002 : 17) ajoute que celle-ci doit se faire dans différents contextes afin que l'élève arrive, comme 1'écrit Giasson (1990:203) «à se construire un portrait plus complexe de la signification de ce mot ». D'autre part, Chabanne et al. (2008: 89) proposent que cette acquisition soit liée à la lecture des textes littéraires et à leur spécificité. David (2000 : 37) rappelle que le développement du lexique s'effectue de façon incidente à la lecture : « elle lui est consubstantielle » et

(3) Les mots actifs sont ceux qu'un individu comprend et utilise spontanément.

(4) Les mots passifs sont ceux qu'un individu comprend de façon plus ou moins précise mais qu'il n'utilise pas. 
le processus d'acquisition est simple : « il correspond à ce que l'on nomme L'effet Matthieu $^{(5)}$ : plus un élève lit et plus il augmente son lexique, et mieux en retour, il comprend les textes qu'on lui propose ce qui accroît encore ses capacités lexicales, et ainsi de suite. \ Certains auteurs (dont Jousse et al., 2008 : 154) affirment que cette acquisition est liée aux tâches d'écriture. Il semblerait donc que l'acquisition du lexique traverse toutes les activités scolaires et qu'elle soit mouvante car comme l'écrit Florin (1993 : 111), «le mot ne constitue pas une entité unifiée, statique, mais il est un matériau composite, qui s'enrichit et se transforme avec le développement de nos connaissances ».

\subsection{Bilan}

La construction de dispositifs didactiques d'apprentissage du lexique à l'école doit prendre en compte la catégorisation, une des bases du système conceptuel de l'enfant et développer une réflexion métalinguistique sur la langue dans des activités de lecture et d'écriture. Comme le rappelle Vigner (1993:202), c'est à l'école d'activer des organisations cognitives (telles que le script ou l'inventaire) afin de construire une compétence lexicale chez l'élève en partant de ses connaissances construites au travers de pratiques sociales, reçues de son entourage. Dans la lignée des travaux de Bruner (1983) et de Vygotski (1933), il est indéniable que ce parcours acquisitionnel ne peut se faire sans un étayage « ajusté » (David, 2000 : 39), l'entourage jouant un rôle essentiel dans l'aide et le soutien aux apprentissages lexicaux (Florin, $2002: 3$ ).

\section{La question du lexique du côté des textes institutionnels}

L'école est le lieu d'apprentissage privilégié, comme le rappelle, en 2006, le socle commun ${ }^{(6)}$ : «Enrichir quotidiennement le vocabulaire des élèves est un objectif primordial, dès l'école maternelle et tout au long de la scolarité obligatoire ». La circulaire du 16 mars 2007 (B.O. n¹2 du 22 mars 2007) préconise que doit être mise en place, dès l'école maternelle, une initiation déjà méthodique au vocabulaire afin de « fixer les outils fondamentaux du langage, accroître et affiner le vocabulaire des élèves qui lui sont confiés, pour en garantir une utilisation sûre et appropriée. ». En 2008, le Bulletin Officiel (B.O. du 19 juin 2008) en reprend les principes en accordant une place importante à l'enseignement du lexique à l'école primaire ${ }^{(7)}$. Il essaie de répondre à trois grandes questions : Pourquoi apprendre du vocabulaire à l'école ? Quel vocabulaire apprendre dès la maternelle? Comment apprendre le vocabulaire?

(5) Cf. Stanovitch, K.E. (1986). « Matthew effect in reading : Some consequences of individual differences in the acquisition of literacy », Reading Research Quarterly, 21 (4) : 360 406.

(6) Le socle commun de connaissances et de compétences a été institué par la loi d'orientation et de programme pour l'avenir de l'École du 23 avril 2005. Il est paru en 2006 au Bulletin Officiel.

(7) Sur 30 pages dans le B.O., le mot «vocabulaire » apparaît 67 fois et « lexique » une seule fois, ce qui donne une moyenne de 2,27 occurrences par page alors que le mot «lecture(s)» ou « relecture » (la lecture étant une grande préoccupation de l'école) n'apparaît que 35 fois. 


\subsection{Les enjeux}

Les documents qui accompagnent les programmes répondent déjà à la première question : «La période qui va de deux à trois ans correspond à un développement important du vocabulaire et il est important de l'accompagner " $\left(\mathrm{MEN}^{(8)}, 2001: 29\right)$ ou encore « Le temps de l'école maternelle correspond, dans le développement de l'enfant, à une période d'explosion lexicale [...] il importe que ce temps soit mis à profit pour aider tous les enfants à élargir leur capital lexical. » (MEN, 2006b : 55). La première raison de cet apprentissage est donc d'ordre développemental, ce qui correspond aux travaux des psycholinguistes. Une seconde raison est apportée par le Bulletin Officiel (2008:12) : «Les enfants apprennent ainsi le vocabulaire (noms, verbes, adjectifs, adverbes, prépositions) qui leur permet non seulement de comprendre ce qu'ils entendent (Qui fait quoi ? À qui ? Où ? Quand? Comment ?), mais aussi d'échanger en situation scolaire, avec efficacité, et d'exprimer leur pensée au plus juste ». Nous retrouvons là l'enjeu mentionné par les chercheurs : la réussite scolaire en réception et en production.

\subsection{Les référentiels}

En réponse à la deuxième question, le Bulletin Officiel (2008:29) donne des repères précis pour les trois sections de la maternelle. Les catégories grammaticales sont explicitement mentionnées. En petite section (PS) : on trouve les noms, les verbes, quelques adjectifs, en moyenne section (MS) et en grande section (GS) : les noms, les verbes, les adjectifs, les adverbes, les comparatifs. Les domaines lexicaux sont aussi spécifiés : en PS, le vocabulaire concerne les actes du quotidien, les activités de la classe, les relations avec les autres; en MS s'ajoute le lexique relatif à l'écrit (aux histoires) et en GS, le vocabulaire des sentiments. L'école maternelle a donc un rôle majeur à jouer dans les apprentissages lexicaux.

\subsection{L'apprentissage}

Concernant la question de l'apprentissage, le Ministère (2008:12) commence par une mise en garde qui rappelle les travaux des chercheurs (dont Fayol, 2006) : « Chaque jour, dans les divers domaines d'activité, et grâce aux histoires que l'enseignant raconte ou lit, les enfants entendent des mots nouveaux, mais cette simple exposition ne suffit pas pour qu'ils les mémorisent ». Puis il apporte une réponse : «L'acquisition du vocabulaire exige des séquences spécifiques, des activités régulières de classification, de mémorisation de mots, de réutilisation du vocabulaire acquis, d'interprétation de termes inconnus à partir de leur contexte. En relation avec les activités et les lectures, l'enseignant veille à introduire chaque semaine des mots nouveaux (en nombre croissant au fil de l'année et d'année en année) pour enrichir le vocabulaire sur lequel s'exercent ces activités ». Nous retrouvons là ce que les chercheurs avaient mentionné : l'accès à la catégorisation, la fréquence et la diversité des rencontres, le lien avec les activités discursives en réception et en production.

(8) MEN ou Ministère de l'Éducation Nationale ( $c f$. bibliographie). 
Mais la lecture des textes officiels ne permet pas aux enseignants de construire des «leçons de mots ${ }^{(9)}$. Nos différentes observations de classes ${ }^{(10)}$, même dans les écoles où le projet ${ }^{(11)}$ était axé sur le lexique, ont conforté l'idée que l'enseignement du vocabulaire n'était pas fondé sur une progression rigoureuse, des séquences spécifiques, des activités systématiques et régulières, une réflexion sur le lexique. Il nous a paru alors nécessaire de répondre aux sollicitations des enseignants de maternelle afin de les aider à didactiser cet enseignement en construisant et en mettant en place un dispositif expérimental.

\section{La conception du dispositif expérimental d'enseignement}

\subsection{Les principes gouvernant le dispositif}

En nous appuyant sur les travaux de recherche et les textes institutionnels, nous avons construit un dispositif qui respecte les principes suivants.

Le premier principe consiste à utiliser des référentiels de mots. Celui du Ministère ${ }^{(12)}$ nous paraît moins adapté que celui de Boisseau (2000), élaboré à partir de différentes sources (échelle Dubois-Buyse ${ }^{(13)}$, imagiers du commerce dont le célèbre Dictionnaire du Père Castor ${ }^{(14)}$...). Ces sources ont été complétées, mises à jour par Boisseau et ses collaborateurs. Ce référentiel répertorie 750 mots à 3 ans, 1.750 mots à 4 ans et 2.750 mots à 5 ans. Il ne s'agit pas de mots nouveaux qu'il faut apprendre comme un empilement de connaissances sur une tabula rasa, mais d'un bagage lexical correspondant à un taux de fréquence et utilisé dans le cadre d'ateliers de classe et de sorties pédagogiques. Ces mots constituent un lexique premier, mais fondamental, en lien avec les expériences, les émotions, l'environnement du jeune enfant.

Le deuxième principe a pour objet de mettre en place un apprentissage méthodique en organisant régulièrement des leçons de mots et en construisant des activités spécifiques. C'est par des situations ritualisées et des rencontres dans différents contextes que l'élève s'approprie et réutilise le vocabulaire nécessaire pour sa pratique future de la langue parlée et écrite. Une des tâches de l'enseignant est de garder des traces du travail effectué autour du mot étudié afin d'évaluer les progrès des élèves et leur évolution sur le chemin de la conceptualisation. Une autre tâche est de constituer un «trésor commun de mots de l'école ${ }^{(15)} »$ afin de garder la mémoire des significations des mots étudiés tout au long de l'année.

(9) Cette appellation: « leçons de mots » apparaît dans la circulaire de mars 2007 et nous la reprenons à notre compte.

(10) Ces observations ont été menées par les deux auteures durant l'année 2008-2009.

(11) Le projet d'école est la pièce maîtresse de la nouvelle politique de l'école, mise en place à la suite de la loi d'orientation du 10 juillet 1989. Il est toujours d'actualité avec la nouvelle loi d'orientation de 2005.

(12) Sur le site du Ministère : http ://www.eduscol.education.fr, on trouve une liste de fréquence des mots mais il s'agit des mots les plus fréquents de la langue écrite française $\left(\mathrm{XIX}^{\mathrm{e}}\right.$ et $\mathrm{XX}^{\mathrm{e}}$ siècles).

(13) Ters, F., Reichenbach, D. et Mayer, G. (1977, $5^{\text {e }}$ édition revue et corrigée, $1^{\text {re }}$ édition en 1940). L'Echelle Dubois-Buyse d'orthographe usuelle française. Paris : O.C.D.L.

(14) Giraud, R. (1999). Le dictionnaire du Père Castor. Paris : Père Castor Flammarion.

(15) Cette expression apparaît dans la circulaire de mars 2007. 
Le troisième principe consiste à aider l'élève à passer du vocabulaire passif au vocabulaire actif afin d'acquérir un lexique plus riche et plus précis. Les rencontres occasionnelles de mots ou la mémorisation systématique et mécanique de listes de mots ne suffisent pas à élargir le lexique de l'enfant, à dépasser celui de sa sphère sociale. En lui offrant l'occasion de s'interroger sur un nombre réduit de mots, l'enseignant aide l'élève, dans des situations d'échanges, à approfondir la connaissance et l'usage de ces mots, dépassant ainsi les limites de ce qu'il peut ou croit connaître. Ces échanges favorisent le développement d'une conscience métalinguistique.

Le quatrième principe vise à établir des relations hiérarchiques entre les mots, c'est-à-dire organiser le lexique par la catégorisation. Cette opération développe chez l'élève des compétences métalexicales qui le conduisent à la conceptualisation, facilitant ainsi le stockage des mots en mémoire, leur mise en lien et leur réutilisation pertinente. Ce travail sur les mots est aussi une aide pour fixer leur forme orthographique.

Le cinquième principe a pour objet d'articuler l'apprentissage du lexique avec des activités de lecture et avec celles de production de textes à l'oral ou à l'écrit. Parce que la lecture des textes, riches et variés ${ }^{(16)}$, permet un contact vivant avec les mots, elle facilite l'accroissement et le réinvestissement du vocabulaire.

Si ces principes sont présentés de façon linéaire dans l'article, ils ne sont pas hiérarchisés dans la pratique de la classe : ils s'imbriquent et s'interpénètrent. Il est évident que pour mettre en œuvre ce dispositif, le rôle de l'enseignant est primordial : il est là pour conduire les activités. Il doit adopter une posture de médiateur, s'appuyant sur le principe d'étayage. Les échanges langagiers autour des mots que l'enseignant contribue à favoriser entre élèves et avec lui vont dans le sens d'une réelle acquisition du lexique.

\subsection{La description du dispositif didactique : le mot de la semaine}

Chaque semaine, un mot (que nous appellerons désormais « mot-vedette») est présenté aux élèves par l'enseignant. Il doit appartenir au référentiel des mots que l'élève doit connaître à la fin de l'école maternelle et/ou avoir un lien avec le projet de classe. Ce mot-vedette peut avoir été découvert lors d'une rencontre hasardeuse au cours d'une activité de langage liée au projet de classe ou dans un album. Le dispositif ritualisé se déroule sur quatre jours d'enseignement par semaine ${ }^{(17)}$ à l'école maternelle.

Le premier jour est consacré à faire émerger les représentations des élèves sur le sens du mot-vedette et à noter par écrit leur production orale. Pour cela, l'enseignante pratique la dictée à l'adulte en écrivant les mots ou les phrases sur une grande affiche. Ces traces sont indispensables pour mesurer l'évolution des acquisitions des élèves.

Lors du deuxième jour les élèves, avec l'aide de l'enseignante, trient leurs

(16) Martinot (2000:92) postule que le type et la qualité des données offertes à l'enfant déterminent son parcours acquisitionnel.

(17) En France, les élèves vont à l'école les jours suivants : lundi, mardi, jeudi et vendredi. 
productions et font apparaître des catégories (définition du mot-vedette, propriétés, actions), notées sur une deuxième affiche. Cette catégorisation entraîne une réflexion sur l'organisation du lexique : synonymes, homonymes, expressions, etc.

Au cours du troisième jour, des ouvrages de littérature de jeunesse (documentaires ou fiction) vont contribuer à modifier ou compléter les premières représentations des élèves. Par exemple, à propos du mot-vedette « soleil », un élève de grande section qui avait proposé : «le soleil il y a des petites planètes dedans » a vu sa définition invalidée par la lecture d'un documentaire. Les textes fictionnels livrent de nouvelles phrases avec le mot-vedette, pris dans plusieurs acceptions, et offrent l'occasion de constituer une anthologie de phrases littéraires recueillies au fil des rencontres.

Le quatrième jour, une affiche du mot-vedette est réalisée comportant l'écriture et le dessin du mot. Ecrire suppose que les élèves fassent un travail d'identification du nombre de syllabes puis de correspondance entre les phonèmes et les graphèmes pour chacune des syllabes. A cela s'ajoute l'identification du genre du mot si c'est un nom ${ }^{(18)}$. Au niveau du signifié, les élèves sélectionnent, parmi toutes les phrases de la semaine, celle(s) qu'ils désirent conserver sur le panneau référent.

Voici deux panneaux référents réalisés en grande section. Le premier, en janvier, autour du mot-vedette « soleil » comporte une phrase d'élève et le second, en juin, autour de «bouquet» renferme une phrase littéraire puis une phrase d'élève :

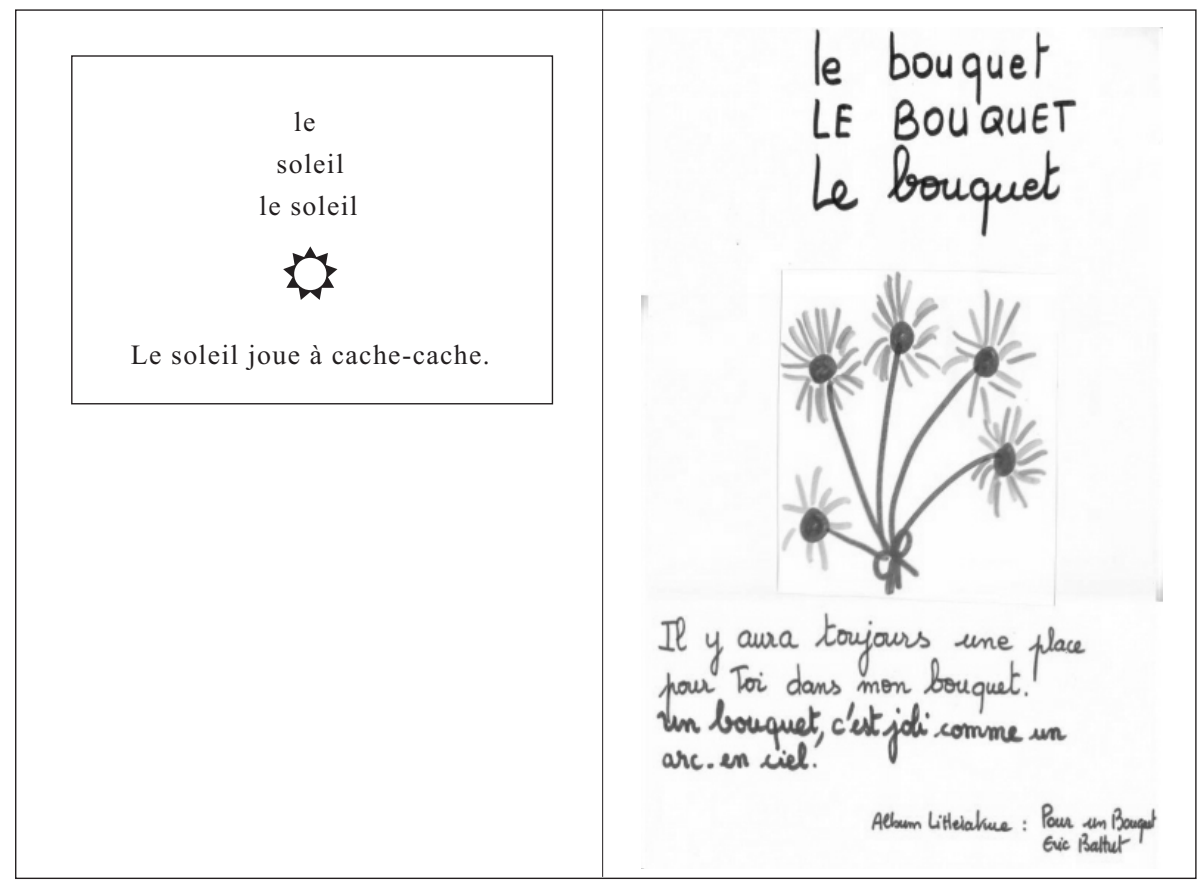

(18) Au fil des mois, un tableau référent du genre des noms se construit et est affiché dans la classe. 
Ces mots vedettes, au fil des mois, viennent s'ajouter à d'autres et enrichissent le dictionnaire de la classe que l'enseignante élabore avec les élèves. Ils prennent sens dans un contexte syntaxique et préparent ainsi les élèves à la mise en mots de l'écriture.

Le dispositif didactique, décrit ci-dessus, rend compte de sa progression dans le temps mais ne présente en aucun cas toutes les activités possibles autour du mot-vedette.

\section{L'expérimentation en grande section}

\subsection{Le contexte}

Ce dispositif didactique a été présenté à plusieurs enseignantes de grande section d'une même circonscription de Marseille au mois d'avril. Quatre d'entre elles ${ }^{(19)}$ se sont portées volontaires pour entrer dans ce projet, deux exerçant avec des publics d'élèves appartenant à un milieu socioculturel favorisé, les deux autres avec un public d'élèves appartenant à un milieu socioculturel moins favorisé. Chacune d'elles a choisi un mot-vedette.

La première enseignante a extrait le mot «soleil» du référentiel du Ministère, conformément au projet d'école. La deuxième a sélectionné le mot «mer » qui s'est imposé naturellement parce qu'elle avait déjà réalisé différentes activités avec ses élèves tout au long de l'année, en lien direct avec la mer : lecture d'un conte sur la mer (en décembre), travail sur les états de l'eau et le cycle de l'eau (en janvier et février), activités autour du sel (en mars), sortie pédagogique à la mer (en avril). La troisième a choisi le mot «bouquet » parce qu'il s'inscrivait dans le projet «patrimoine» de la classe et renvoyait à la visite de jardins (de l'école en septembre, d'un jardin au bord de la mer en octobre) et à la rencontre d'œuvres picturales (visite de l'exposition Van Gogh à Marseille en novembre). La quatrième a centré son travail sur le mot «kangourou » rencontré lors de la lecture d'un album ${ }^{(20)}$. Les données orales et écrites de ces quatre classes autour des mots vedettes constituent notre corpus d'exploitation ${ }^{(21)}$.

Ces quatre mots se retrouvent dans la liste établie par Boisseau pour la grande section. Néanmoins ce n'est pas le rang de fréquence du mot qui importe; ce sont les activités que l'enseignante va conduire dans le dispositif autour du mot-vedette dans le but de l'inscrire dans le vocabulaire actif de l'élève grâce aux manipulations opérées sur la langue à partir de ce mot.

(19) Nous tenons à remercier les enseignantes qui ont expérimenté notre dispositif dans leur classe et qui nous ont apporté une aide précieuse par leurs remarques.

(20) Chapman, J. (2003). Ça sert à quoi, un kangourou? Namur : Mijade.

(21) Les exemples oraux ne seront pas ponctués sauf si cela est indispensable pour une meilleure lecture. L'élève sera identifié par E (Elève) suivi d'un numéro (le numéro de la prise de parole dans le corpus du mot-vedette) et l'enseignante sera identifiée par M. 


\subsection{Analyse des résultats de l'expérimentation}

Ce dispositif a fourni aux enseignantes un cadre de travail précis avec des activités structurées et répétitives ${ }^{(22)}$. Il leur a permis d'adopter une démarche reposant sur les cinq principes énoncés ( $c f$. supra) : partir des représentations des élèves, catégoriser, valider ou invalider les hypothèses par les confrontations et la réflexion métalinguistique. Il a contribué à faire évoluer les représentations des élèves, à les aider à organiser leur lexique et à les faire entrer dans la grammaire : ce sont ces trois points que nous allons développer.

\subsubsection{Une évolution des représentations des élèves}

La première question posée pour faire émerger les représentations sur le motvedette était une interrogation en «Qu'est-ce que... ? », reformulée le plus souvent par l'enseignante : «Un bouquet c'est?», «On dit qu'est-ce que c'est un kangourou?», «La mer c'est quoi ? », «Qu'est-ce que c'est qu'un soleil ? ». Ces reformulations sont nécessaires pour aider les élèves à s'exprimer : elles constituent un véritable étayage, comme le rappelle David (2000).

Ce moment de langage oral autour des représentations sur le mot-vedette a été l'occasion pour les élèves d'interagir pour préciser la pensée de l'autre (ex. 1 et ex. 2), la compléter (ex. 3), la modifier (ex. 4) comme dans les exemples suivants :

(1) M : Qu'est-ce qu'un soleil?

E8 : il y a des petites planètes dedans

E9 (s'adressant à l'enseignante) : il croyait qu'il y avait la planète terre dedans

(2) M : Qu'est-ce qu'un bouquet?

E13 : c'est beau

E14 : c'est pour faire beau

(3) M : Qu'est-ce qu'un bouquet?

E8 : j'ai offert à ma maman un bouquet

E9 : pour la fête des mamans

(4) M : Qu'est-ce qu'un kangourou?

E2: il a une pochette pour mettre son bébé dedans

E3 : c'est pas une pochette c'est une poche

L'évolution des représentations premières des élèves est le fruit d'activités métalinguistiques et d'activités de lectures conduites par l'enseignante par le jeu des interactions et des confrontations dans la classe. Ces co-constructions participent à l'intégration du lexique et à sa mémorisation.

Cette évolution aboutit à la construction d'une définition collective des mots vedettes à la fin de la semaine, inscrite sur une affiche par dictée à l'adulte. Le dispositif permet ainsi de faire entrer les élèves de maternelle dans une tâche complexe de production écrite que représente l'écriture d'une définition, comme le montrent les deux exemples suivants (ex. 5 et ex. 6) :

(22) Branca-Rosoff et al. (2008: 266) prônent également un enseignement du vocabulaire à travers des activités spécifiques et une certaine répétitivité. 
(5) M : Qu'est-ce qu'un bouquet?

Des représentations des élèves le premier jour:

E4 : je pense à un bouquet de violettes

E11 et E12: c'est des fleurs

E18: c'est des fleurs qui sentent bon

Définition proposée par les élèves en fin de semaine:

un bouquet c'est beaucoup de fleurs mises ensemble, c'est des fleurs groupées

(6) M : Qu'est-ce que la mer?

Des représentations des élèves le premier jour :

E3 : c'est de l'eau

E4 : c'est comme la neige qui fond qui devient de l'eau

E5 : c'est comme un grand lac

Définition proposée par les élèves en fin de semaine:

la mer est une grande étendue d'eau salée

Dans ce dernier exemple, la définition retenue par les élèves est celle du dictionnaire. Elle correspond à un besoin de la classe d'avoir une définition partagée par tous. Face aux différentes propositions émises, les élèves ont demandé à l'enseignante de les aider à trouver un référent commun. Celle-ci a introduit le dictionnaire, les préparant ainsi à l'utilisation de cet outil. Le mot «étendue »a été mimé d'un geste avec la main bien à plat pour représenter une grande surface. La lecture de cette définition a emporté tous les suffrages, a augmenté encore leur lexique avec le mot «étendue »; c'est celle que les élèves ont retenue pour l'affiche de la classe :

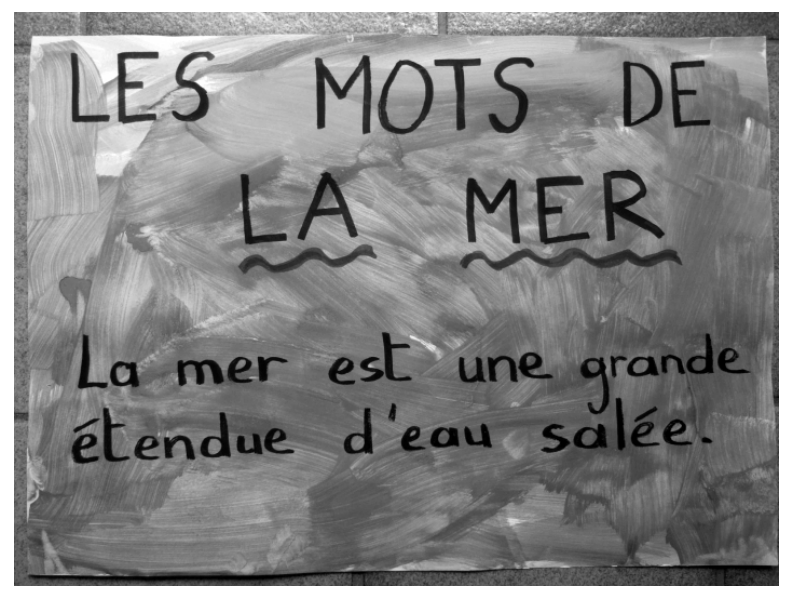

Cet exemple montre que la mise en place de ce dispositif a dépassé ce que nous avions prévu, entraînant la consultation par des élèves de maternelle d'un ouvrage de référence aussi complexe que le dictionnaire.

\subsubsection{Un lexique qui s'organise}

Le dispositif a amené les élèves à catégoriser grâce aux questions posées par l'enseignante. Si la question en «Qu'est-ce que...? ? les a incités à rassembler les énoncés qui pouvaient construire une définition du mot, comme nous l'avons vu précédemment, le recours aux questions en «Comment est... ? » et en «Que 
fait... ? » les a conduits à regrouper des phrases dont le sémantisme renvoyait à des attributs et à des actions. Les attributs produits par les élèves étaient sur le plan grammatical des adjectifs (sauf pour « bouquet» où sont apparus des groupes nominaux prépositionnels, $c f$. affiche ci-dessous : de soleils, de nuit). Pour les actions, les élèves ont recouru à des verbes. Des procédures de substitution les ont amenés à catégoriser les mots en noms, verbes et adjectifs, termes métalinguistiques introduits par l'enseignante. L'ouverture sur trois classes grammaticales a contraint les élèves à ne pas se centrer sur le nom, prédominant dans le développement du langage (Bassano, 2005) ou dans les outils didactiques ${ }^{(23)}$ et à passer de la référence simple (celle des noms) à la prédication et à la grammaire, comme l'écrit David (2000 : 34). Les élèves ont ainsi utilisé ce métalangage grammatical qui a pris sens à partir des manipulations sur la langue. Ils ont approché ces différents concepts et ont constitué des référentiels sur des affiches, comme dans l'exemple de « bouquet» :

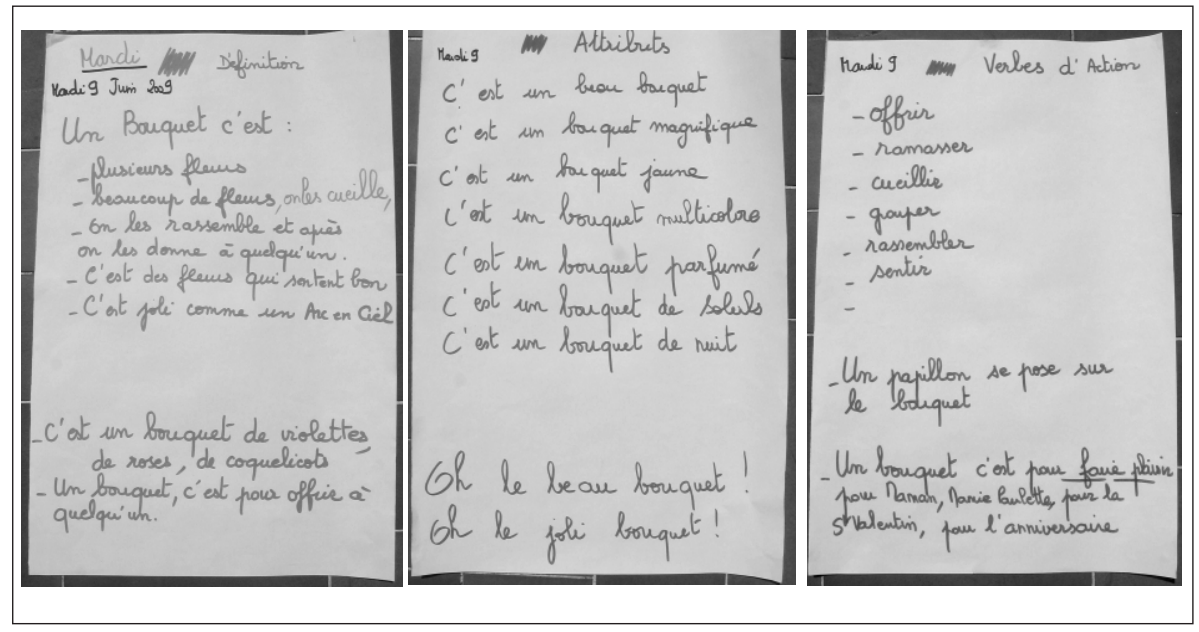

La recherche dans les textes littéraires ou documentaires a amené les élèves à compléter leurs listes de mots, à augmenter leur stock lexical d'adjectifs comme pour le mot «mer» (forte, déchaînée, plate, infinie...) ou de verbes (scintille, étincelle, ondule, danse...). Afin de fixer le sens de quelques mots nouveaux, l'enseignante, qui a travaillé sur le mot «mer», a choisi d'associer le signifiant et le signifié, liant ainsi l'activité d'écriture à une activité plastique, comme dans cet exemple où l'écriture des mots reproduit l'effet de la mer déchaînée ${ }^{(24)}$ :

(23) Duvignau et Garcia-Debanc (2008: 22) remarquent que la prédominance des noms se manifeste même dans les outils parascolaires d'apprentissage du lexique disponibles dans le commerce : "Les outils d'apprentissage des verbes sont sous-représentés par rapport à ceux destinés à l'apprentissage des noms. En effet, il existe une très grande quantité d'imagiers et d'abécédaires consacrés exclusivement aux noms. »

(24) Lors de l'exposition de ces écritures de mots, l'enseignante a dû préciser sur une affiche destinée aux parents : «Ecriture déformée volontairement » afin que ceux-ci ne jugent pas la qualité de l'écriture mais comprennent le sens de cette écriture déformée. 


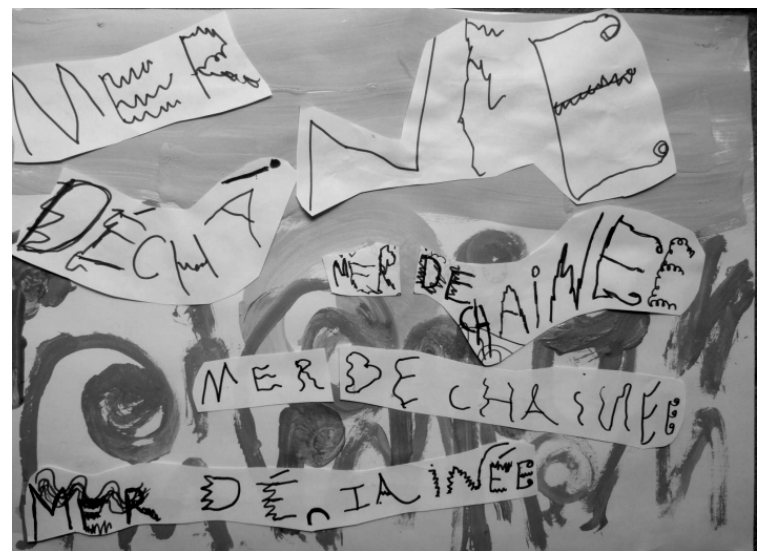

En fonction du mot-vedette, d'autres questions se sont ajoutées aux trois premières : "Qui vit dans la mer ? » ou encore « Où vit le kangourou ? " permettant de constituer des inventaires (listes d'animaux et de végétaux marins ou lieux de vie de l'animal) participant à de nouvelles catégorisations, tel ce panneau regroupant les animaux vivant dans la mer :

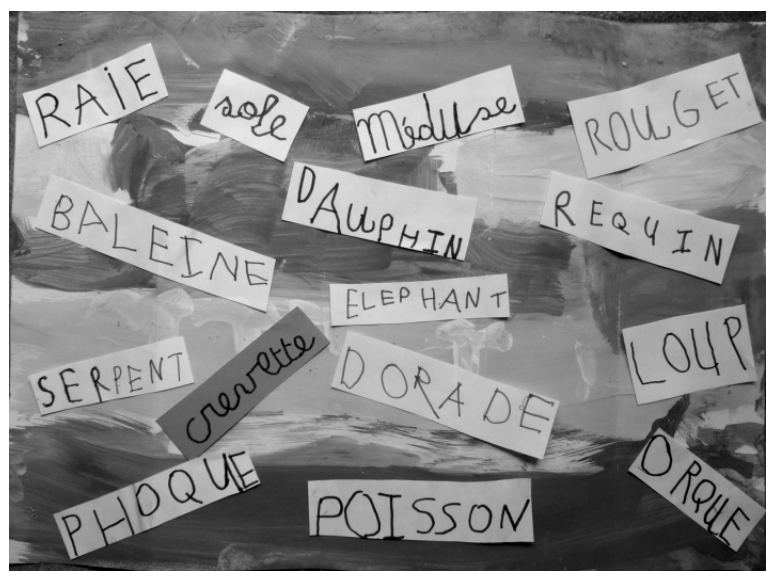

Chaque nom rencontré a ainsi pu être reclassé dans une autre catégorie, prouvant aux élèves que le lexique s'organise différemment selon l'entrée qu'on privilégie.

Le questionnement sur le mot-vedette « kangourou »a été l'occasion pour l'enseignante de faire entrer les élèves dans une démarche de lecteur, les contraignant à développer une stratégie de chercheur d'informations :

(7) M : Où vit le kangourou?

E21 : il vit en Amérique

E22 : non il vit en Australie

E23 : il vit aussi en Afrique

E24: au zoo

M : Où peut-on chercher l'information pour se mettre d 'accord? 
E25: dans un livre qui parle de la nature

E26 : à la télé

E27 : sur l'ordinateur

E28: sur Internet

E29 : dans un documentaire

Les élèves se sont alors engagés dans l'appropriation d'un environnement documentaire (numérique ou non) pour valider les réponses exactes concernant le lieu de vie du kangourou.

Ces questions et ces inventaires, parce qu'ils constituent des procédés de traitement et de stockage de l'information ${ }^{(25)}$, facilitent le lien entre le développement lexical de l'enfant et ses progrès catégoriels. L'opération de catégorisation initiée par le dispositif a contraint les élèves à brasser les mots, à les associer différemment et à créer de nouvelles catégories.

Ce sont toutes ces activités autour du mot-vedette, toutes ces recherches, ce questionnement qui ont amené les élèves à approcher les familles de mots : «mer / marin », à cerner la notion de termes génériques : " animaux marins ", "végétaux marins", à travailler sur les nuances de sens et à graduer le lexique ( «gelée» $v s$ « glacée», « froide» $v s$ «fraiche»). Lorsqu'un élève a défini le mot « mer» comme «c'est une maman », l'enseignante en a profité pour attirer l'attention de la classe sur le rôle joué par l'orthographe en français et ainsi les a sensibilisés au problème d'homophonie. Avec le mot « kangourou », les élèves ont réactivé le sens des adjectifs «sauvage » et « domestique » ${ }^{(26)}$ et la confrontation de ces mots les a conduits à approcher la notion d'antonymie.

Nul doute que ces manipulations sur le lexique, ces regroupements au fil des séances ont un effet facilitateur sur les inférences en lecture et en écriture des élèves.

\subsubsection{Un lien entre lexique et grammaire}

Ce dispositif didactique expérimental a permis aux enseignantes de prendre conscience que le plus important, ce n'est pas la quantité de mots qu'elles offrent à leurs élèves mais la qualité des connaissances lexicales qu'elles construisent avec eux. C'est ce que constate David (2000:37) quand il écrit que les élèves apprennent beaucoup de mots, mais ne parviennent guère à les utiliser à bon escient.

Au-delà des procédés de stockage et de traitement d'unités isolées, le travail sur le lexique a fait entrer les élèves dans la grammaire par la mise en mots que ces activités ont suscitée, ce qui va dans le sens des recherches de Bassano (2005) : le développement grammatical est dans une relation de dépendance par rapport au développement lexical, à condition qu'un certain stock lexical ait été constitué. C'est ainsi que les verbes produits isolément pour «bouquet » ont été mis en mots dans un petit scénario dont Bruner (1983) a montré l'importance pour l'acquisition du langage. Cette saynète, mimée et parlée par les élèves, illustre la

(25) C'est ce qu'écrit Vigner (1993 : 201) à propos du script et de l'inventaire.

(26) La notion d'animaux domestiques avait été construite lors de la visite à la ferme et celle d'animaux sauvages lors de lecture d'albums (animaux vivant dans la jungle). 
cueillette des fleurs : je cueille..., je groupe..., je lie..., je sens..., j'offre... De la même façon, à partir de la liste de verbes pour le mot «kangourou » (grandir, manger, sauter, faire des bonds, s'occuper de son bébé), les élèves ont produit des phrases qu'ils ont enchaînées dans un script mis en scène. Le dispositif a ainsi permis le lien entre lexique, grammaire et écriture et l'entrée des mots dans le vocabulaire actif des enfants.

Les échanges réflexifs induits par le dispositif tout au long de la semaine ainsi que les regroupements de mots ont conduit les élèves à construire des familles de mots : ce fut le cas lors de la production des dérivés comme mer / marin, soleil / ensoleillé, fleur / fleuri.

En copiant les verbes sur le panneau intitulé «Que fait la mer?», les élèves se sont aperçus que les étiquettes ne comportaient qu'un seul mot : étincelle, gronde, bouge... sauf pour : est en colère. Par cette observation mise en débat dans la classe, ils ont approché la spécificité du verbe être qui se construit ici dans une collocation ${ }^{(27)}$.

Lors des séances sur le mot «bouquet», ils ont produit eux-mêmes des collocations ${ }^{(28)}$ («bouquet de senteurs », « bouquet à la fin du feu d'artifice», «bouquet de couleurs »), des comparaisons (« un bouquet c'est comme un arc-enciel »). Ils ont aussi pratiqué des associations telle que refléter et miroir (« la mer est bleue parce que le ciel se reflète dans l'eau, la mer est comme un miroir »). Avec les textes littéraires, ils ont approché la métaphore (« Il y aura toujours une place pour toi dans mon bouquet»). Ces structures plus ou moins figées offrent aux élèves un contexte syntaxique qui donne sens au mot et facilite son entrée en mémoire.

Quant au mot «mer », il a été l'occasion pour les élèves de rencontrer des expressions comme : «ce n'est pas la mer à boire», de les comprendre et de les utiliser en les intégrant à leur langage :

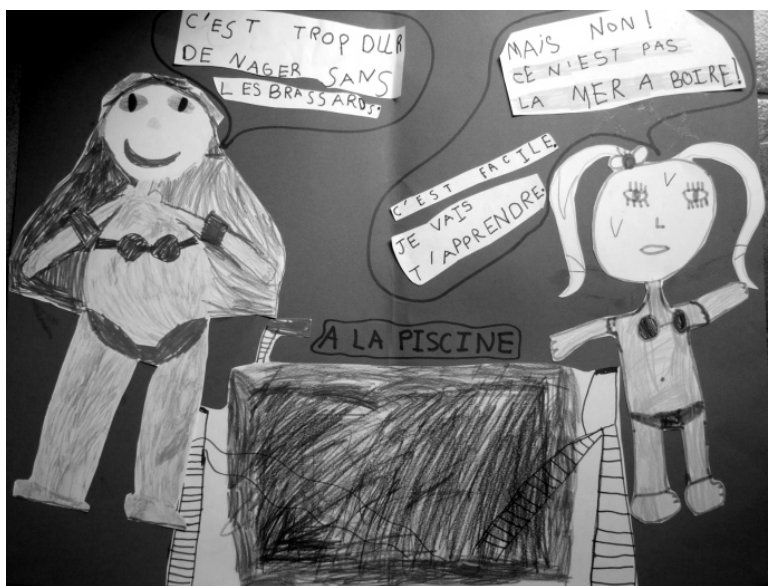

(27) Le mot « collocation » désigne l'assemblage lexical entre « être » et sa valence « en colère $»$.

(28) Grossmann et al. (2008) ou Novakova et Bouechava (2008) proposent d'enseigner aux élèves natifs ou aux jeunes apprenants les mots et collocations associées. 
Ces diverses activités ont amené ces jeunes élèves à manipuler la langue pour en comprendre son fonctionnement et ainsi établir des liens entre lexique et grammaire, favorisant par là-même la structuration de la pensée et la mise en mots.

\section{Conclusion}

Cette étude sur la mise en place d'un dispositif didactique pour un enseignement structuré du lexique dès l'école maternelle nous a permis de constater que l'évolution des représentations de tous les élèves, quel que soit leur milieu socioculturel, va non seulement dans le sens d'une amélioration du lexique mais participe aussi à la mise en place d'opérations cognitives transférables à l'appropriation de tout nouveau mot.

La fixation des mots en mémoire afin qu'ils soient compris ou utilisés en production par les élèves dépend de la répétitivité des activités pour classer, brasser, mémoriser le vocabulaire lors des phases de manipulations, d'observations, et de débats co-constructifs. C'est parce que les situations d'énonciation proposées sont riches et variées que les élèves étendent d'une part leur répertoire lexical et d'autre part développent des compétences métalinguistiques. L'apport des textes littéraires et documentaires est propice au développement de ces compétences.

Le dispositif proposé a fait du mot-vedette un déclencheur, entraînant les élèves à dépasser l'unité mot pour l'inscrire dans une unité de sens, à lier le lexique à la grammaire lors d'activités de production (de scénarios, de collocations, d'expressions...). Par cette mise en mots, le dispositif les a sensibilisés à toutes les richesses qu'offre la langue et les a fait entrer dans l'écriture.

Au-delà de l'acquisition du lexique, le dispositif expérimental a tenté de construire chez l'élève un comportement de « questionneur de mots », que nous espérons voir se manifester sans la présence de l'enseignant. 


\section{Références}

BASSANO, D. (2005) : «Production naturelle précoce et acquisition du langage : l'exemple du développement des noms », Lidil, 31, 61-84.

BOCH, F. (2004) : «Apprentissage du lexique et production d'écrits : une articulation féconde? », In E. Falardeau (Ed.), Le français : discipline singulière, plurielle ou transversale? Actes du $9^{\circ}$ Colloque international de 1'AIRDF, 26-28 août 2004 [CD-ROM]. Québec : AIRDF.

BOISSEAU, P. (2000) : Introduction à la pédagogie du langage-Version abrégée. Rouen : CRDP de Haute Normandie.

BrancA-Rosoff, S., Reboul-Touré, S. et PAGnier, T. (2008) : « Linguistique de corpus et didactique du lexique : la mise en mots des sensations dans une classe de CE1 ». In F. Grossmann \& S. Plane (Eds.), Les apprentissages lexicaux. Lexique et production verbale (pp. 253-273). Villeneuve d'Ascq : Presses Universitaires du Septentrion.

BRUNER, J. (1983/1987) : Comment les enfants apprennent à parler. (J. Piveteau et J. Chambert, trad.). Paris : Retz. (Ouvrage original publié en 1983, New York).

CAlaque, E. (2004) : « Construction du vocabulaire et construction des connaissances au cours moyen ». In E. Falardeau (Ed.), Le français : discipline singulière, plurielle ou transversale? Actes du $9^{\mathrm{e}}$ Colloque international de l'AIRDF, 26-28 août 2004 [CD-ROM]. Québec : AIRDF.

Chabanne, J-C., Cellier, M., Dreyfus, M. et Soulé, Y. (2008) : « Les enjeux lexicaux de la lecture littéraire ». In F. Grossmann \& S. Plane (Eds.), Les apprentissages lexicaux. Lexique et production verbale (pp. 85-101). Villeneuve d'Ascq : Presses Universitaires du Septentrion.

DAVID, J. (2000) : «Le lexique et son acquisition : aspects cognitifs et linguistiques », Le Français aujourd'hui, 131, 31-41.

DREYFUS, M. (2004) : «Pratiques et représentations de l'enseignement du lexique à l'école primaire ». In E. Falardeau (Ed.), Le français : discipline singulière, plurielle ou transversale? Actes du $9^{\text {ème }}$ Colloque international de l'AIRDF, 26-28 août 2004 [CD-ROM]. Québec : AIRDF.

DUVIGNAU, K. et GARCIA-DEBANC, C. (2008) : «Un apprentissage du lexique verbal par proximité sémantique : quand la représentation lexicale facilite la tâche d'écriture ». In F. Grossmann \& S. Plane (Eds.), Les apprentissages lexicaux. Lexique et production verbale (pp. 17-41). Villeneuve d'Ascq : Presses Universitaires du Septentrion.

FAYOL, M. (2006) : «L'orthographe et son apprentissage ». In Enseigner la langue : orthographe et grammaire. Les journées de l'Observatoire National de la Lecture, mars 2006 (pp. 53-73). Paris : ONL.

FLORIN, A. (1993) : «Les connaissances lexicales des enfants d'école primaire». Repères, $8:$ : 93-112.

- (2002, 3 avril) : Le développement du lexique et l'aide aux apprentissages. Conférence prononcée dans le cadre des réunions organisées par la Commission départementale Maîtrise de la Langue et du Langage de SeineSaint-Denis, Académie de Créteil.

GiAsson, J. (1990) : La compréhension en lecture. Bruxelles : De Boeck. 
GOMBERT, J-E. (1990) : Le développement métalinguistique. Paris : PUF.

Grossmann, F., B och, F. et CAVAlla, C. (2008) : «Quand l'écriture n'empêche pas les sentiments... Quelques propositions pour mieux intégrer la dimension lexicale ». In F. Grossmann \& S. Plane (Eds), Les apprentissages lexicaux. Lexique et production verbale (pp.191-218). Villeneuve d'Ascq : Presses Universitaires du Septentrion.

Jousse, A-L., Polguère, A. et Tremblay, O. (2008) : « Du dictionnaire au site lexical pour l'enseignement/apprentissage du vocabulaire ». In F. Grossmann \& S. Plane (Eds.), Les apprentissages lexicaux. Lexique et production verbale (pp. 141-157). Villeneuve d'Ascq : Presses Universitaires du Septentrion.

LAHIRE, B. (1993) : Culture écrite et inégalités scolaires. Lyon :Presses Universitaires de Lyon.

LIEURY, A. (2003) : « Mémoire et apprentissages scolaires », revue de didactologie des langues-cultures, 2003/2, 130, 179-186.

MARTINOT, C. (2000) : «Etude comparative des processus de reformulation chez des enfants de 5 à 11 ans », Langages, 140, 92-123.

MINISTÈRE DE L'EDUCATION NATIONALE ET MINISTÈRE DE L'ENSEIGNEMENT SUPÉRIEUR ET DE LA RECHERCHE. (2008) : B.O. Horaires et programmes d'enseignement de l'école primaire. Hors série n³ du 19 juin 2008.

- (2007) : B.O. Mise en ouvre du socle commun de connaissances et des compétences : l'acquisition du vocabulaire à l'école primaire. Encart B.O. $\mathrm{n}^{\circ} 12 \mathrm{du} 22$ mars 2007.

- (2006a) : B.O. Socle commun de connaissances et de compétences. Encart B.O. $n^{\circ} 29$ du 20 juillet 2006.

MINISTÈRE DE LA JEUNESSE, DE L'EDUCATION NATIONALE ET MINISTÈRE DE LA RECHERCHE. (2006b) : Le langage à l'école maternelle. Document d'accompagnement des programmes. Paris : CNDP.

- (2001) : Pour une scolarisation réussie des tout-petits. Document d'accompagnement des programmes. Paris : CNDP.

Novakova, I. et BoueChava, E. (2008) : «Les collocations du type avoir ou être + Nom de sentiment en français et en russe. Aspects linguistiques et didactiques ». In F. Grossmann \& S. Plane (Eds.), Les apprentissages lexicaux. Lexique et production verbale (pp. 219-232). Villeneuve d'Ascq : Presses Universitaires du Septentrion.

PICOCHE, J. (1992) : Précis de lexicologie française. L'étude et l'enseignement du vocabulaire. Paris : Nathan.

VIGNER, G. (1993) : «Le monde, les mots et l'école : Eléments d'une didactique du vocabulaire à l'école élémentaire ». Repères, 8 : 191-209.

Vygotski, L. (1933/1997) : Pensée et Langage. (F. Sève, trad.). Paris : La Dispute. 